\title{
Erratum: Probing multistrange dibaryons with proton-omega correlations in high-energy heavy ion collisions [Phys. Rev. C 94, 031901(R) (2016)]
}

\author{
Kenji Morita $\odot$, Akira Ohnishi, Faisal Etminan, and Tetsuo Hatsuda
}

(Q) (Received 25 November 2019; published 20 December 2019)

DOI: $10.1103 /$ PhysRevC.100.069902

The original paper is found to contain a typographical error in the binding energy of $p \Omega$ with the $V_{\mathrm{II}}+$ Coulomb potential. The binding energy value of $6.3 \mathrm{MeV}$ shown in Table I of the original paper should be corrected to be $0.63 \mathrm{MeV}$. The conclusions and other results are not affected.

TABLE I. Revised version of Table I in the original paper. The binding energy $\left(E_{\mathrm{B}}\right)$, the scattering length $\left(a_{0}\right)$, and the effective range $\left(r_{\text {eff }}\right)$ with and without the Coulomb attraction in the $p \Omega$ system. Physical masses of the proton and $\Omega$ are used.

\begin{tabular}{llccr}
\hline \hline \multicolumn{2}{c}{ Spin-2 $N \Omega$ Potentials } & $V_{\mathrm{I}}$ & $V_{\text {II }}$ & $V_{\text {III }}$ \\
\hline \multirow{3}{*}{ Without Coulomb } & $E_{\mathrm{B}}(\mathrm{MeV})$ & & 0.05 & 24.8 \\
& $a_{0}(\mathrm{fm})$ & -1.0 & 23.1 & 1.60 \\
& $r_{\text {eff }}(\mathrm{fm})$ & 1.15 & 0.95 & 0.65 \\
\hline \multirow{3}{*}{ With Coulomb } & $E_{\mathrm{B}}(\mathrm{MeV})$ & & 0.63 & 26.9 \\
& $a_{0}(\mathrm{fm})$ & -1.12 & 5.79 & 1.29 \\
& $r_{\text {eff }}(\mathrm{fm})$ & 1.16 & 0.96 & 0.65 \\
\hline \hline
\end{tabular}

